Received: 2018.10.24

Accepted: 2018.12.04

Published: 2019.04.18
Authors' Contribution: Study Design A Data Collection B Statistical Analysis C Data Interpretation D Manuscript Preparation E Literature Search F Funds Collection $G$

\section{Anthecotulide Sesquiterpene Lactone Exhibits Selective Anticancer Effects in Human Malignant Melanoma Cells by Activating Apoptotic and Autophagic Pathways, S-Phase Cell Cycle Arrest, Caspase Activation, and Inhibition of NF- $\kappa B$ Signalling Pathway}

ABDEF Chenyang Li

ACDEFG Xiuping Han
Department of Dermatology, Shengjing Hospital of China Medical University, Shenyang, Liaoning, P.R. China
Corresponding Author: Source of support:
Xiuping Han, e-mail: PierceDlag@yahoo.com

Departmental sources
Background: We examined the anticancer potential of anthecotulide against SK-MEL-24 malignant melanoma cells. The apoptotic and autophagic effects of anthecotulide were also investigated.

Material/Methods: The cell viability of SK-MEL-24 human malignant melanoma cells was evaluated by WST-1 assay. Fluorescence microscopy using acridine orange and ethidium bromide staining, as well as Western blot analysis, were used to study apoptotic effects induced by anthecotulide. Autophagy was assessed by Western blot analysis and fluorescence microscopy. Effects of anthecotulide on cell cycle progression were analyzed by flow cytometry.

Results: The results revealed that anthecotulide exerts significant growth-inhibitory effects on SK-MEL-24 cells. The IC of anthecotulide against the SK-MEL-24 cells was found to be $10 \mu \mathrm{M}$. However, the anticancer effects against the normal cells were minimal (IC50; $100 \mu \mathrm{M})$. Investigation of the underlying mechanism revealed that anthecotulide prompts apoptotic cell death of the SK-MEL-24 cells, which was linked with increased expression of Bax and decreased expression of Bcl-2. It also triggered concentration-dependent activation of caspase 3 and 9. Anthecotulide induced autophagy in the SK-MEL-24 cells, which was associated with upregulation of LC3 II and Beclin-1 expression. Anthecotulide also halted the SK-MEL-24 cells at S-phase of the cell cycle and downregulated the expression of Cyclin B1. However, the expression of p27 was upregulated.

Conclusions: These results indicate anthecotulide is a potent lead molecule for the treatment of melanoma. In vivo and other related experiments are warranted to further assess this promising drug candidate.

MeSH Keywords: $\quad$ Apoptosis • Autophagy • Cell Cycle • Melanoma

Full-text PDF: https://www.medscimonit.com/abstract/index/idArt/913771 


\section{Background}

Sesquiterpene lactones (SQLS) are a diverse and large group of natural metabolites found across the plant kingdom, and approximately 5000 SQLs have been reported so far from plants [1]. Over the years, SQLs have received considerable attention owing to their potent bioactivities, such as anticancer and anti-microbial action [2,3]. A number of SLs have been reported to exhibit significant anticancer activity and many SQLS are currently undergoing clinical trials [4]. Anthecotulide is an important SQL, but there have been few studies of the anticancer activity of anthecotulide [5]. The present study was designed to examine the anticancer effects of anthecotulide on human malignant melanoma cells. Melanoma is a serious cancer and accounts for about $4 \%$ of all cancer-related deaths. It is the sixth most common type of cancer in the United States, where it has been reported that 1 in every 5 individuals develop skin cancer during their lifetime [6]. There is strong evidence that exposure to ultraviolet radiation initiates the development of melanoma and much attention has recently been focused on exploring the potential of natural products to protect against UVR-induced skin cancer [7]. The treatment of melanoma is generally difficult due its late diagnosis, as well as metastasis to distant organs. Moreover, the insufficient effectiveness of chemotherapeutic drugs, associated adverse effects, and the emergence of drug resistance further make it difficult to treat diseases such as cancer [8]. Therefore, the development of more effective and safer chemotherapy from natural sources holds great promise. Herein, for the first time, we report that anthecotulide inhibits the growth of human SKMEL-24 malignant melanoma cells. The anticancer effects of anthecotulide are mainly due to the induction of apoptosis, accompanied with alteration of the $\mathrm{Bax} / \mathrm{Bcl}-2$ ratio and caspase activation. Further, anthecotulide induced autophagy in the SK-MEL-24 cells along with upregulation of LC3 II protein levels. Anthecotulide also concentration-dependently induced the $S$ phase arrest of SK-MEL-24 cells. Several signal transduction pathways are activated in cancer cells, such as NF- $\mathrm{KB}$ signalling cascade [9]. Herein, we found that anthecotulide inhibits this pathway, indicative of the potent anticancer activity of anthecotulide. To conclude, anthecotulide has potent anticancer activity and may prove useful for the development of chemotherapy for melanoma.

\section{Material and Methods}

\section{Cell lines and culture conditions}

The human melanoma cell line SK-MEL-24 cells and the normal HaCat cells were obtained from the Cancer Research Institute of Beijing (Beijing, China) and maintained in Dulbecco's modified Eagle's medium (Invitrogen Life Technologies, MA) supplemented with $10 \%$ fetal bovine serum (Invitrogen Life Technologies, MA), $100 \mu \mathrm{g} / \mathrm{ml}$ streptomycin, and $100 \mathrm{U} / \mathrm{ml}$ penicillin $\mathrm{G}$ (Himedia, $\mathrm{PA}$ ) in an incubator at $37^{\circ} \mathrm{C}$ with $5 \% \mathrm{CO}_{2}$.

\section{Cell viability assay}

The effect of anthecotulide on the viability of the SK-MEL-24 and $\mathrm{HaCat}$ cells was assessed by WST-1 assay. In brief, the melanoma cells were cultured at a density of $2.5 \times 10^{5}$ cells/well in 96-well plates and subjected to treatment with varied concentrations ( 0 to $200 \mu \mathrm{M}$ dissolved in dimethyl sulfoxide) of anthecotulide. The control cells were treated with only $2 \%$ DMSO. This followed by the incubatation of the SK-MEL-24 cells with WST-1 for $3 \mathrm{~h}$ at $37^{\circ} \mathrm{C}$, and the proliferation rate was determined by assessing absorbance at $450 \mathrm{~nm}$ using a UV spectrophotometer (Thermo Fisher, Waltham, MA).

\section{Acridine orange and ethidium bromide double staining for the detection of apoptosis}

For AO/EB staining, the SK-MEL-24 cells were grown in 6-well plates $\left(0.6 \times 10^{6}\right.$ cells/well). Following incubation for $12 \mathrm{~h}$, the SKMEL-24 cells were subjected to anthecotulide treatment $(0,5,10$, and $20 \mu \mathrm{M})$ for $24 \mathrm{~h}$ at $37^{\circ} \mathrm{C}$. As the cells sloughed off, $25-\mu \mathrm{l}$ cell cultures were put onto glass slides and subjected to staining with a solution of AO (acridine orange) and EB (ethidium bromide). The slides were then covered with a covers lip and examined with a fluorescence microscope (Thermo Fisher, Waltham, MA).

\section{GFP-LC3 transfection for the detection of autophagy}

For detection of autophagy, the SK-MEL-24 cells were grown to $70 \%$ confluence and transfected with GFP-LC3 plasmids using Lipofectamine 2000 (Invitrogen) according to the manufacturer's guidelines. The transfected cells were then treated with various concentrations of anthecotulide $(0,5,10$, and $20 \mu \mathrm{M})$ for $24 \mathrm{~h}$ and subsequently monitored by fluorescence microscopy (Thermo Fisher, Waltham, MA).

\section{Cell cycle analysis}

SK-MEL-24 melanoma cells were incubated with various concentrations of anthecotulide $(0,5,10$, and $20 \mu \mathrm{M})$ for $24 \mathrm{~h}$. The cells were then subjected to washing with phosphatebuffered saline (PBS). Then, the SK-MEL-24 cells were stained with propidium iodide ( $\mathrm{PI}$ ) and the distribution of the cells in cell cycle phases was assessed by use of a FACS flow cytometer (BD Biosciences, San Jose, CA).

\section{Western blot analysis}

To determine the expression of the selected proteins in the anthecotulide-treated $(0,5,10$, and $20 \mu \mathrm{M})$ malignant 


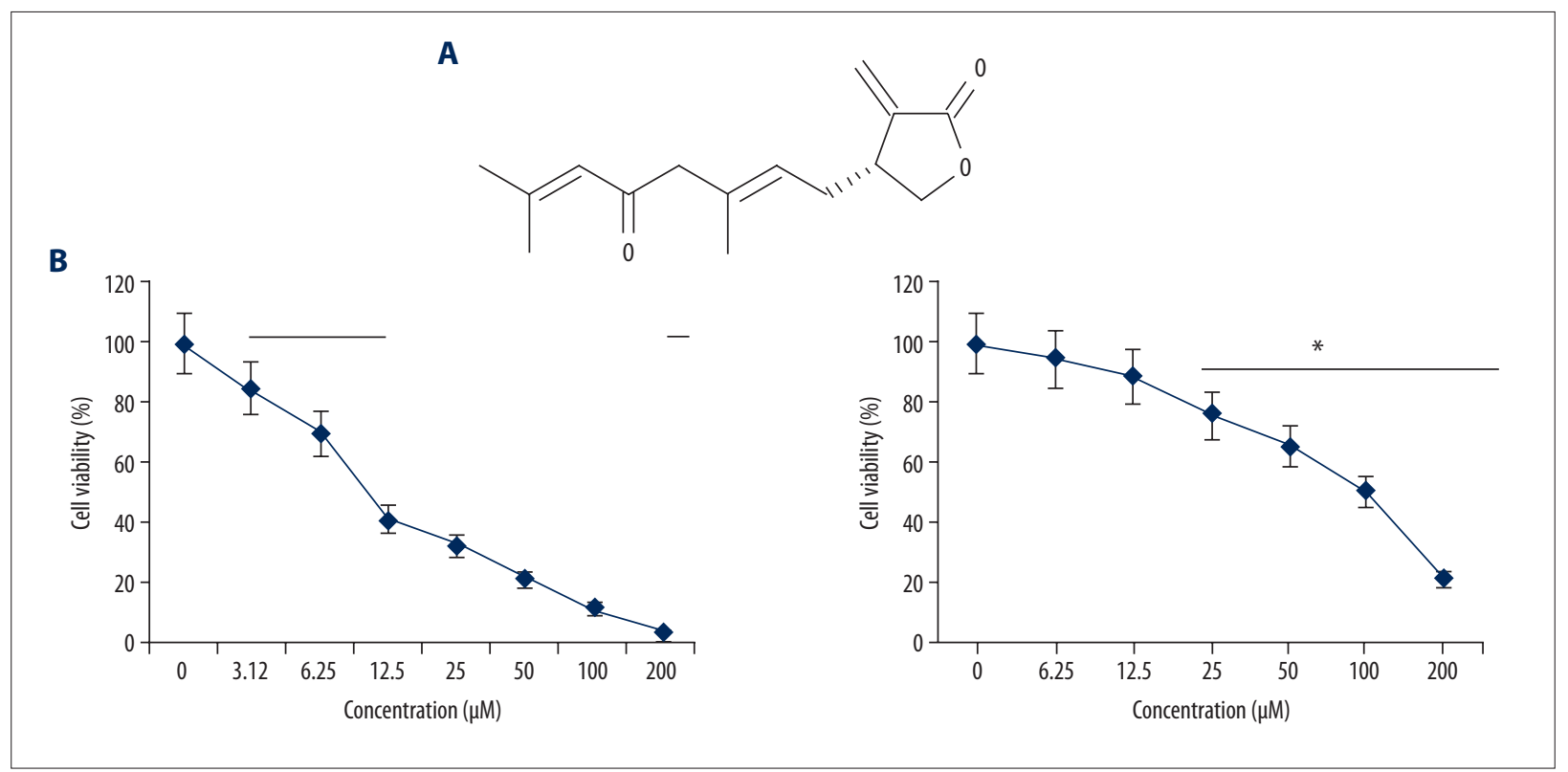

Figure 1. (A) Structure of anthecotulide. (B) WST-1 assay showing the effects of anthecotulide on the viability of the SK-MEL-24 melanoma and $\mathrm{HaCat}$ normal cells. The experiments were performed in triplicate and results are shown as mean \pm SD $(p<0.05)$.

melanoma cells, the cells were lysed with RIPA buffer and the protein contents of lysates were estimated by BCA assay. The samples were then loaded on the SDS-PAGE. The gels were then transferred to nitrocellulose membranes and subjected to treatment with primary antibody at $4^{\circ} \mathrm{C}$ for $24 \mathrm{~h}$. After this, the membranes were incubated with HRP-conjugated secondary antibody for $50 \mathrm{~min}$ at $25^{\circ} \mathrm{C}$. Enhanced chemiluminescence reagent was used to visualise the protein bands.

\section{Statistical analysis}

The experiments were performed in triplicate. The values represent mean \pm SD. $P<0.05$ was regarded as statistically significant. We used the $t$ test and GraphPad Prism 7 for statistical analysis.

\section{Results}

\section{Anthecotulide inhibits exerts antiproliferative effects on SK-MEL-24 melanoma cells}

The anti-proliferative effects of anthecotulide (Figure $1 \mathrm{~A}$ ) on the malignant SK-MEL-24 melanoma cells were examined by WST-1 assay. It was found that that anthecotulide exerts antiproliferative effects on the SK-MEL-24 melanoma cell line and exhibited an $\mathrm{IC}_{50}$ of $10 \mu \mathrm{M}$ (Figure $1 \mathrm{~B}$ ), but the anticancer effects of anthecotulide against the normal HaCat cells were minimal $\left(\mathrm{IC}_{50} ; 100 \mu \mathrm{M}\right)$. In addition, we found that the anticancer effects of anthecotulide on the melanoma cells occurred in a dose-dependent manner.

\section{Anthecotulide triggers apoptosis in SK-MEL-24 melanoma cells}

The apoptosis-inducing effects of anthecotulide on the malignant melanoma SK-MEL-24 cells were investigated by AO/EB staining. The results of AO/EB assay showed that anthecotulide induced apoptotic cell death in the SK-MEL-24 melanoma cells (Figure 2). Analysis of the protein expression of the apoptosis biomarker proteins revealed that anthecotulide increased the expression of Bax and decreased the expression of $\mathrm{Bcl}-2$. Furthermore, anthecotulide also concentration-dependently triggered activation of caspase 3 and 9 in the SK-MEL-24 cells (Figure 3).

\section{Anthecotulide triggers autophagy in SK-MEL-24 melanoma cells}

We also investigated whether anthecotulide induces autophagy in SK-MEL-24 cells. LC3 II was transiently overexpressed in the SK-MES-4 cells by transfecting the cells with transient pEGFP-LC3 plasmid and then cells were treated with various concentrations of anthecotulide. We found that anthecotulide caused a concentration-dependent increase in the GFP-LC3 punctate dots in the SK-MEL-24 cells, as indicated by fluorescence microscopy (Figure 4). This indicates that anthecotulide induced autophagy in SK-MEL-24 cells. For the confirmation of autophagy, the expression of autophagy-associated proteins was examined, showing that asiaticoside caused an increase in levels of Beclin-1 and LC3-II, but no effects were observed on the expression of LC3-I (Figure 5). 


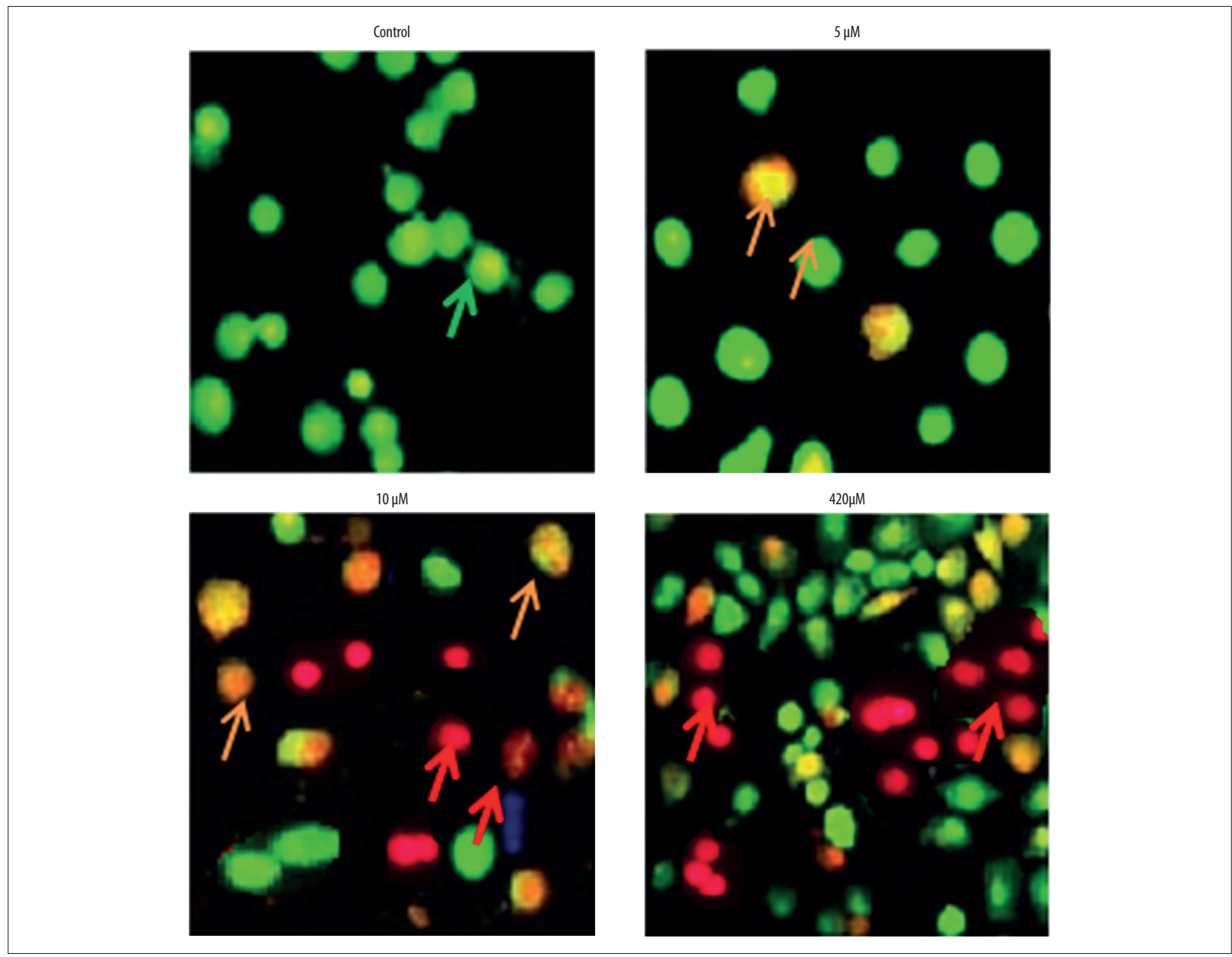

Figure 2. AO/EB staining showing the induction of apoptosis in SK-MEL-24 melanoma cells at indicated concentrations.

The experiments were performed in triplicate. Green arrows depict normal cells, yellow arrows depict early apoptosis, and the red arrows depict late apoptosis.

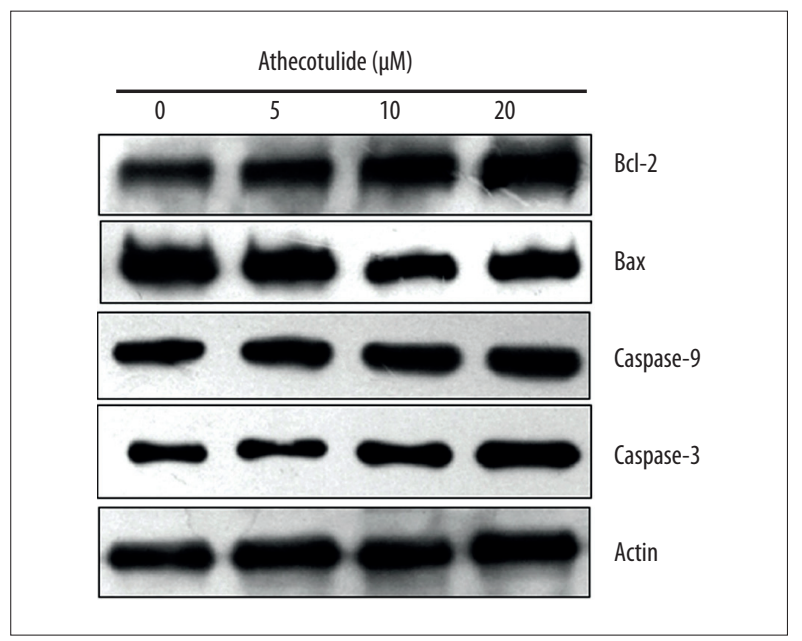

Figure 3. Bax, Bcl-2, and Caspase 3 and 9 expressions after treatment with anthecotulide at indicated concentrations, as depicted by Western blot analysis. The experiments were performed in triplicate.

\section{Anthecotulide causes the S phase cell cycle arrest of SK- MEL-24 melanoma cells}

The effects of anthecotulide on the distribution of SK-MEL-24 melanoma cells (SK-MEL-24) in various cell cycle phases were assessed by flow cytometry, showing that Anthecotulide caused a remarkable increase in the percentage of SK-MEL-24 melanoma cells in the $S$ phase of the cell cycle. The percentage of SK-MEL-24 esophageal cancer cells in the $S$ phase increased from $15.6 \%$ to $32.3 \%$ upon treatment with anthecotulide (Figure 6). These results clearly indicate that anthecotulide induces S phase cell cycle arrest of SK-MEL-24 melanoma cells. Moreover, S phase cell cycle arrest of SK-MEL-24 cells by anthecotulide was also associated with concentration-dependent suppression of Cyclin B1 and upregulation of p27 expression (Figure 7). 


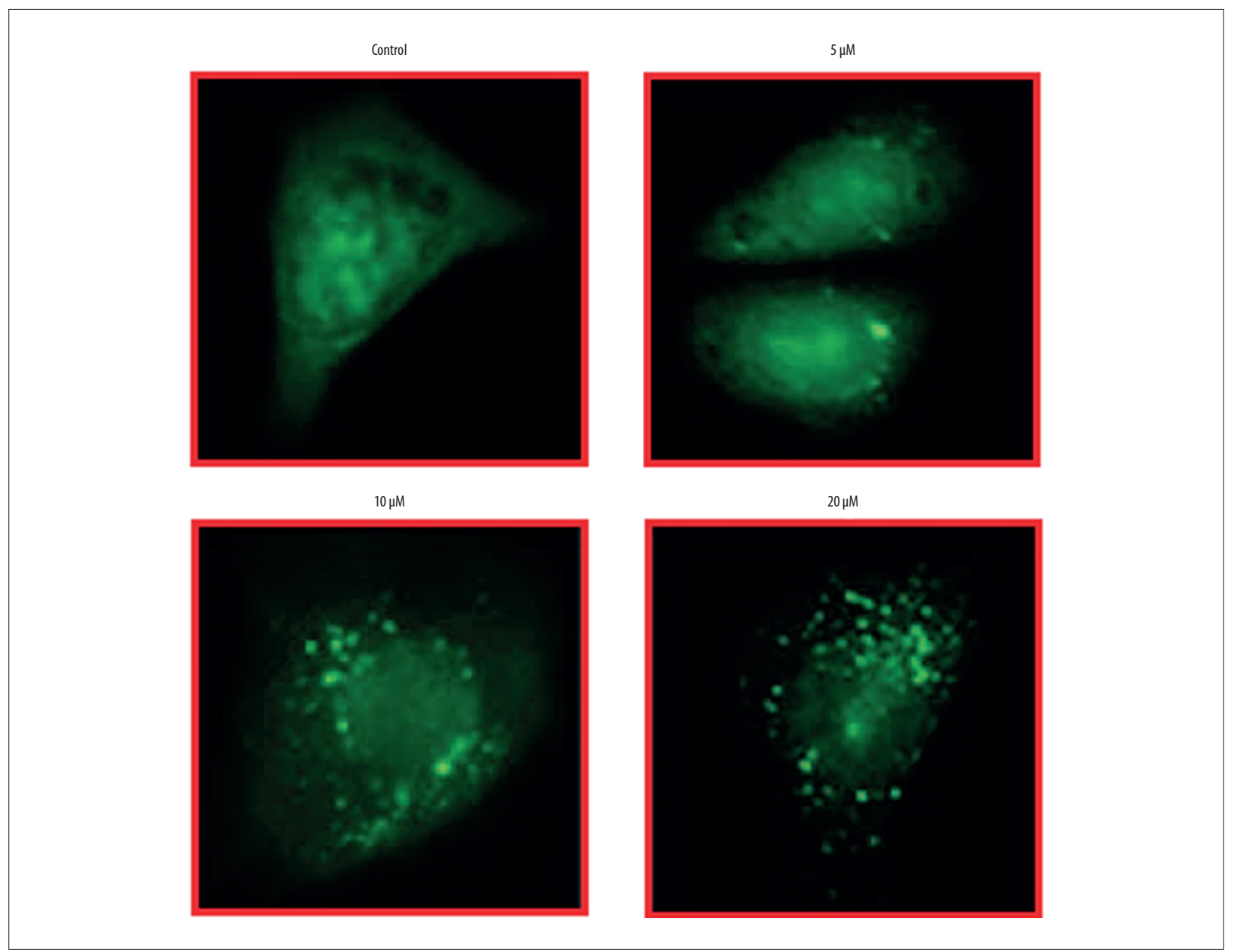

Figure 4. Effect of anthecotulide on the expression of LC3 II in PGL-FL3 transfected cells, as indicated by fluorescence microscopy. The experiments were performed in triplicate.

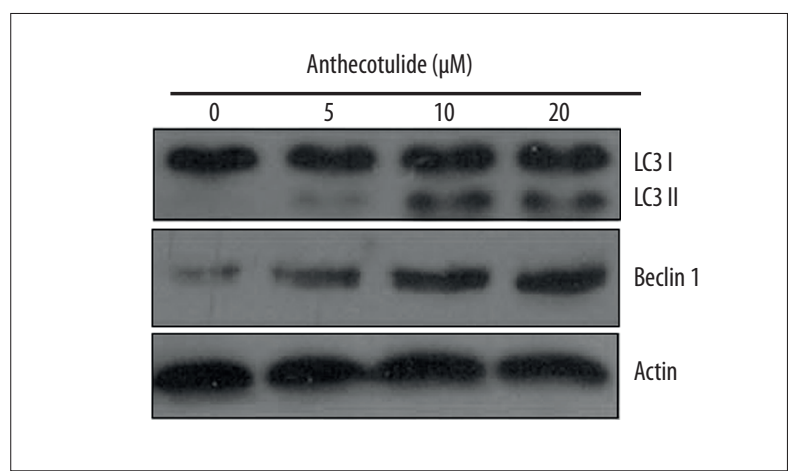

Figure 5. LC3 I and II and Beclin-1 expression after treatment with anthecotulide at indicated concentrations, as depicted by Western blot analysis. The experiments were performed in triplicate.

\section{Anthecotulide inhibits the NF- $\kappa B$ signalling pathway}

It has been reported that many signalling pathways are activated in cancer cells and these pathways are considered important targets for anticancer drugs. The effect of anthecotulide was also investigated on the NF- $\mathrm{\kappa B}$ signalling pathway in SK-MEL-24 cells. We found that anthecotulide causes a large decrease in the expression of the NF- $\kappa B$ proteins and this effect was concentration-dependent (Figure 8).

\section{Discussion}

Melanoma is a deadly cancer accounting for approximately $4 \%$ of all cancer-related deaths [7]. The lack of effective drugs, late diagnosis, and emergence of multi-drug resistance makes management of melanoma complicated [10]. In the present study we investigated the anticancer effects of a natural SQL, anthecotulide, on SK-MEL-24 melanoma cells. We found that anthecotulide concentration-dependently suppressed the proliferation of SK-MEL-24 melanoma cells and showed an $\mathrm{IC}_{50}$ of $10 \mu \mathrm{M}$. Although anticancer activities have not been reported previously for anthecotulide, numerous studies have reported the anticancer effects of natural SQLs; for example, parthenolide 


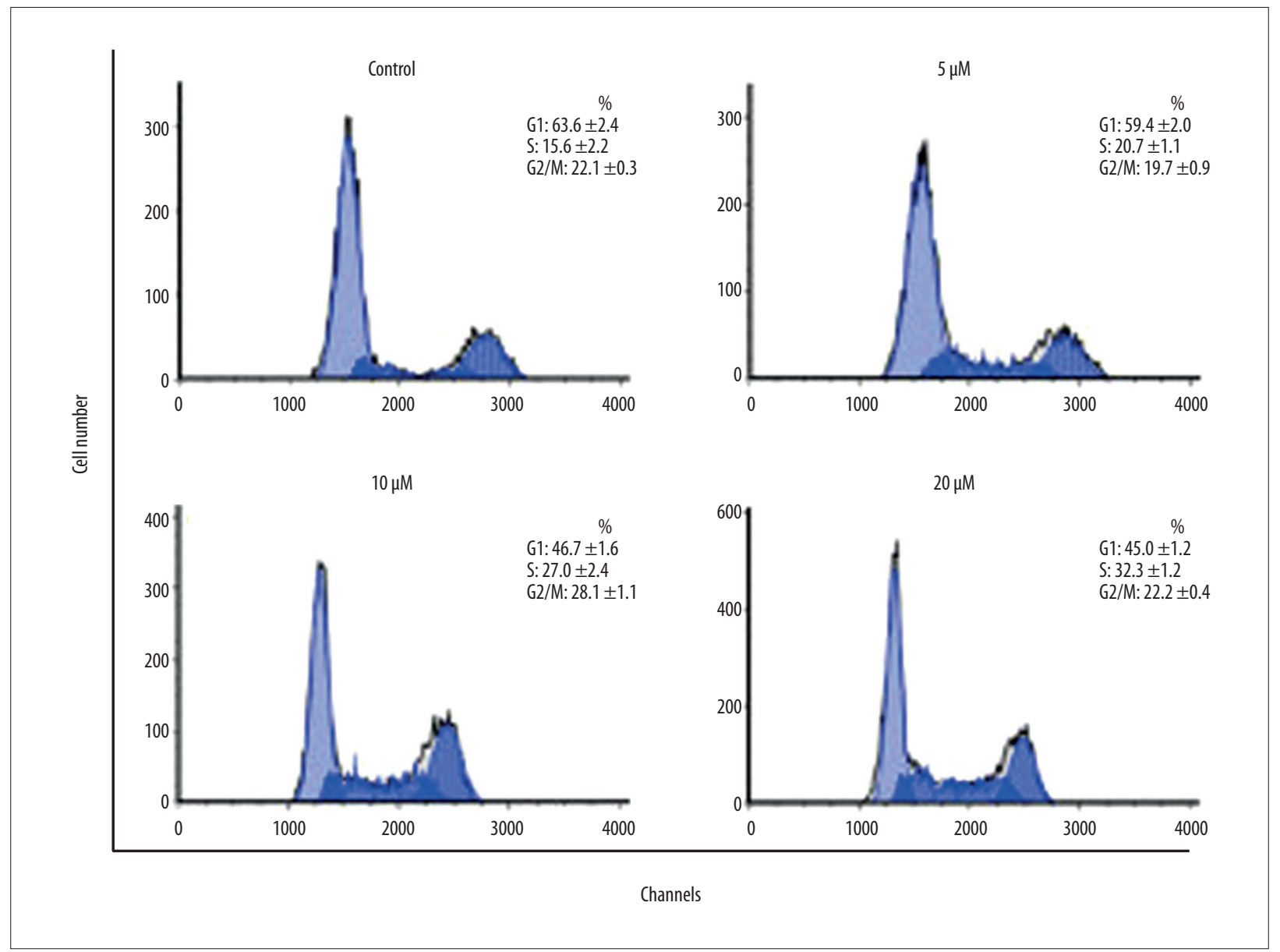

Figure 6. S phase cell cycle arrest as depicted by flow cytometery at indicated concentrations of anthecotulide. The experiments were performed in triplicate.

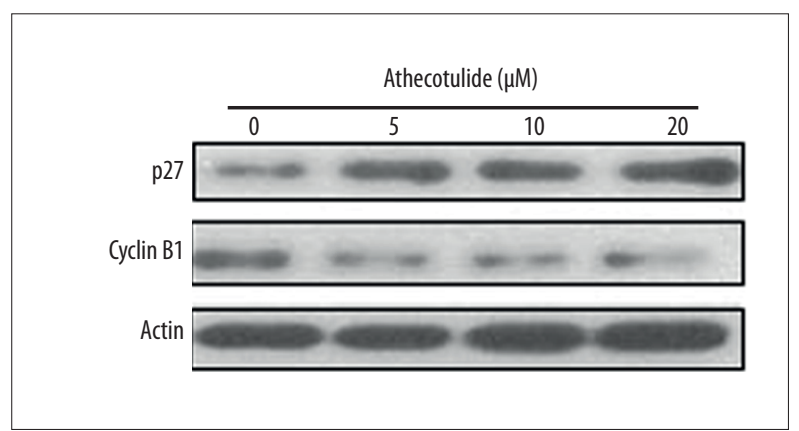

Figure 7. Cyclin B and p27 expression after treatment with anthecotulide at indicated concentrations as depicted by Western blot analysis. The experiments were performed in triplicate.

has been reported to inhibit the growth of cancer cells [11]. Similarly, a sesquiterpene lactone, costunolide, has been reported to halt the growth of MCF-7 cells [12]. Several of the SQLs have been reported to induce autophagy in cancer cells; for example, helenalin has been reported to induce autophagy in cancer cells [13]. Here, we found that anthecotulide induced

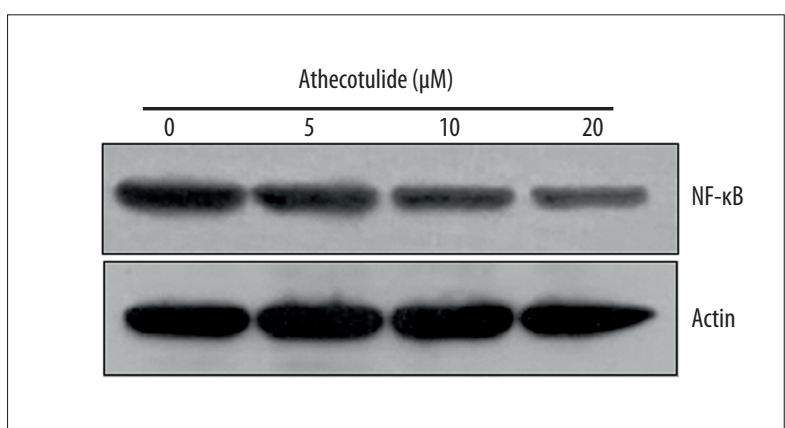

Figure 8. Effect of anthecotulide on NF- $\mathrm{kB}$ expression at indicated concentrations as depicted by Western blot analysis. The experiments were performed in triplicate.

autophagy in SK-MEL-4 cells, along with upregulation of the LC3 II and Beclin 1 expression. SQLs have also been shown to induce apoptosis in cancer cells, which one of the important mechanisms maintaining homeostasis in human tissues [14]. For example, parthenolide has been shown to trigger apoptotic cell death of human acute myelogenous leukemia cells [15]. We found that anthecotulide also triggered apoptosis in the 
SK-MEL-24 cells and was linked with upregulation of Bax and downregulation of $\mathrm{Bcl}-2$ as well as the activation of caspase 3 and 9. Cell cycle arrest is another important mechanism by which anticancer molecules exert their effects [16] and here we found that anthecotulide triggered the $\mathrm{S}$ phase arrest of SKMEL-24 melanoma cells, which was associated with suppression of cyclin B1 and upregulation of p27 expressions. A previous study showed that a SQL, a parthenolide, exerts anticancer effects by inhibiting NF- $\mathrm{\kappa B}$ [17]. Therefore, we also examined the effects of anthecotulide on NF-KB protein expression and found that anthecotulide concentration-dependently inhibited the expression of $\mathrm{NF}-\mathrm{KB}$, indicative of its anticancer potential.

\section{References:}

1. Zhang S, Won YK, Ong CN, Shen HM: Anti-cancer potential of sesquiterpene lactones: Bioactivity and molecular mechanisms. Curr Med Chem Anticancer Agents, 2005; 5(3): 239-49

2. Rodriguez E, Towers GH, Mitchell JC: Biological activities of sesquiterpene lactones. Phytochemistry, 1976; 15(11): 1573-80

3. Lee KH, Huang ES, Piantadosi C et al: Cytotoxicity of sesquiterpene lactones. Cancer Res, 1971; 31(11): 1649-54

4. Ghantous A, Gali-Muhtasib H, Vuorela $\mathrm{H}$ et al: What made sesquiterpene lactones reach cancer clinical trials? Drug Discov Today, 2010; 15(15-16): 668-78

5. Meyer A, Zimmermann S, Hempel B, Imming P: Anthecotulide: purification, analytical data, absence from chamomile preparations, stability and reactivity, and anti-infective testing. J Nat Prod, 2005; 68(3): 432-34

6. Robert C, Long GV, Brady B et al: Nivolumab in previously untreated melanoma without BRAF mutation. N Engl J Med, 2015; 372(4): 320-30

7. Robert C, Karaszewska B, Schachter J et al: Improved overall survival in melanoma with combined dabrafenib and trametinib. N Engl J Med, 2015; 372(1): 30-39

8. Guy GP Jr., Zhang Y, Ekwueme DU et al: The potential impact of reducing indoor tanning on melanoma prevention and treatment costs in the United States: An economic analysis. J Am Acad Dermatol, 2017; 76(2): 226-33

9. Dolcet X, Llobet D, Pallares J, Matias-Guiu X: NF- $\mathrm{KB}$ in development and progression of human cancer. Virchows Arch, 2005; 446(5): 475-82

\section{Conclusions}

Anthecotulide inhibits the growth of malignant melanoma cells by induction of autophagy and apoptosis. It also induces $S$ phase cell cycle arrest and inhibited the NF- $\kappa B$ signalling pathway, suggesting its potential in the management of melanoma. Further investigations are warranted.

\section{Conflict of interest}

None.

10. Van Allen EM, Miao D, Schilling B et al: Genomic correlates of response to CTLA4 blockade in metastatic melanoma. Science, 2015; 350(6257): 207-11

11. Mathema VB, Koh YS, Thakuri BC, Sillanpää M: Parthenolide, a sesquiterpene lactone, expresses multiple anti-cancer and anti-inflammatory activities. Inflammation, 2012; 35(2): 560-65

12. Bocca C, Gabriel L, Bozzo F, Miglietta A: A sesquiterpene lactone, costunolide, interacts with microtubule protein and inhibits the growth of MCF-7 cells. Chem Biol Interact, 2004; 147(1): 79-86

13. Lim CB, Fu PY, Ky N et al: NF-KB p65 repression by the sesquiterpene lactone, Helenalin, contributes to the induction of autophagy cell death. BMC Complement Altern Med, 2012; 12(1): 93

14. Lowe SW, Lin AW: Apoptosis in cancer. Carcinogenesis, 2000; 21(3): 485-95

15. Guzman ML, Rossi RM, Karnischky L et al: The sesquiterpene lactone parthenolide induces apoptosis of human acute myelogenous leukemia stem and progenitor cells. Blood, 2005; 105(11): 4163-69

16. Hua F, Li CH, Chen XG, Liu XP: Daidzein exerts anticancer activity towards SKOV3 human ovarian cancer cells by inducing apoptosis and cell cycle arrest, and inhibiting the Raf/MEK/ERK cascade. Int J Mol Med, 2018; 41(6): 3485-92

17. Oka D, Nishimura K, Shiba M et al: Sesquiterpene lactone parthenolide suppresses tumor growth in a xenograft model of renal cell carcinoma by inhibiting the activation of NF-KB. Int J Cancer, 2007; 120(12): 2576-81 